Much less is known about transmission of plant mitochondria, in part because its complex and structurally labile genome has not provided the kind of easily assayed RFLP markers available for the plastome. Like the Sequoia report, the few studies available are based on small samples of progeny, but they suggest that maternal inheritance of the mitochondrial genome predominates even in taxa with paternal or biparental transmission of plastids. Before the Sequoia report, there were hints that the rules of mitochondrial transmission were no more absolute than those of the plastid, as some paternal transmission of mtDNA had been observed in barley-rye intergeneric crosses $^{11}$, and biparental inheritance of mitochondrial RNA had been reported in alfalfa ${ }^{12}$. Nevertheless, the finding of strict paternal inheritance of mtDNA among all of the dozen or so progeny analysed in two intraspecific crosses of redwood is news indeed. As the authors themselves point out, the available evidence does not rule out some maternal transmission. Much larger sample sizes would be needed to exclude completely this possibility. But for the first time a species has been found in which it is clear that elimination mechanisms must be postulated for maternal, not paternal mitochondria.

The mitochondrial genomes of animals have received far more attention than those of plants. Animal mtDNA has been characterized as having 'strict' maternal inheritance ${ }^{13}$, although, as in plants, data are available for only a limited array of taxa. Furthermore, the possibility of low levels of paternal leakage cannot be excluded, as there have been remarkably few exhaustive studies of mtDNA inheritance. The apparent rarity of heteroplasmy had long been taken as evidence for maternal inheritance, but even now, when reports of heteroplasmy are commonplace $^{13}$, most examples do not suggest biparental transmission. In virtually all cases, coexisting mitochondrial genomes differ by single changes, often in copy number of tandemly repeated sequences. Heteroplasmy can thus be explained by mutations within homogeneous populations of mtDNA molecules and subsequent drift in genotype frequencies within cell lineages.

In contrast, two recent studies report instances of heteroplasmy in which individual organisms carry two mtDNA genotypes that differ substantially in nucleotide sequence (ref. 14; and W. M. Brown, personal communication). This clearly suggests some paternal mitochondrial transmission in taxa otherwise showing maternal inheritance, and leads to the suspicion that organelle transmission 'rules' may be no more absolute for animals than for plants.

Inheritance patterns are also influenced by changes in the proportions of mtDNA genotypes in germ-cell lineages. Heteroplasmic ancestors will eventually give rise to a group of homoplasmic descendants as a result of random fixation of alternative types. The number of generations required for fixation appears to be very different in the few organisms for which such data are available ${ }^{15-17}$, so it may prove difficult to develop generalizations about intracellular population genetics. For one thing, too few of the parameters, such as effective population size and mutation rate, are known for all but a few taxa. Differential replication will also affect the proportions of two or more organelle types among descendants of a heteroplasmic individual. The success of particular organelle genomes may in part be intrinsic, for example, smaller mtDNA molecules may win the race to replicate ${ }^{16}$. But the close cooperation necessary between organelle and nuclear genomes virtually guarantees that such explanations are likely to be at best only part of a more complex story.

The transmission genetics of organelle DNA has become of critical interest to systematists and evolutionary biologists who wish to trace gene genealogies to gain insight into organism phylogenies. Organelle genomes were originally attractive because of their apparent simplicity, uniparental inheritance and absence of recombination, providing 'non-anastomosing maternal lineages' for the interpretation of patterns of variation in natural populations. The Sequoia evidence joins the increasing body of exceptions to the rule of maternal inheritance. Although natural instances of organelle genome recombination remain unproven, it will surely not be too much of a surprise to find that organelle lineages anastomose as well.

Richard G. Harrison and Jeff J. Doyle are in the Division of Biological Sciences, Cornell University, Ithaca, New York 14853, USA.

1. 'Birky, C.W. Jr A. Rev. Genet. 12, 471-512 (1978).

2. Neale, D.B., Marshall, K.A. \& Sederoff, R.R. Proc. natn. Acad. Sci. U.S.A. 86, $9347-9349$ (1989)

3. Neale, D.B., Wheeler, N.C. \& Allard, R.W. Can. J. For. Res. 16, 1152-1154 (1986).

4. Szmidt, A.E., Alden, T. \& Hallgren, J.-E. Plant molec. Biol. 9, 59-64 (1987).

5. Neale, D.B. \& Sederhoff, R.R. Theor. appi. Genet. 77 $212-216$ (1989).

6. Stine, M., Sears, B.B. \& Keathley, D.E. Theor. appl. Genet. 78, 768-774 (1989)

. Corriveau, J.L. \& Coleman, A.W. Am. J. Bot. 75, 14431458 (1988).

8. Sears, B. B. Plasmid 4, 233-255 (1980).

9. Chiu, W.-L., Stubbe, W. \& Sears, B.B. Curr. Genet. 13, 181-189 (1987).

10. Schumann, C.M. \& Hancock, J.F. Theor. appl. Genet. 78 $863-866$ (1989).

11. Soliman, K., Fedak, G. \& Allard, R.W. Genome 29, 867 872 (1987).

12. Fairbanks, D.J., Smith, S.E. \& Brown, J.K. Theor. appl. Genet. 76, 619-622 (1988).

13. Harrison, R.G. Trends Ecol. Evol. 4, 6-11 (1989).

14. Satta, Y. et al. Genet. Res. 52, 1-6 (1988)

15. Solignac, M., Generment, J., Monnerot, M. \& Mounolou, J.-C. Molec. Gen. Genet. 197, 183-188 (1984).

16. Rand, D.M. \& Harrison, R.G. Genetics 114, 955-970 (1986)

17. Ashley, M., Laipis, P.J. \& Hauswirth, W.W. Nucleic Acids Res. 17, 7325-7331 (1989).

\section{Steering the snow}

SNow, says Daedalus, is a mixed blessing. A snowy landscape is lovely to look at, and highly profitable for the ski-ing industry, but snow is a major nuisance on sportsgrounds, racecourses, runways, and roads. It has to be melted away with massive dressings of salt - which does damage of its own, by crumbling concrete and rusting vehicles. Now Daedalus plans to tame this unruly form of winter weather.

Any brute-force attempt to melt or hold back or pre-precipitate a snowstorm is, of course, doomed to failure; atmospheric energies are far too great. Daedalus's 'Snow-steerer' accepts whatever snow nature provides, and merely steers it in the most convenient direction.

It exploits the phenomenon of the Rayleigh disc, that neat device which detects a sound wave by turning at rightangles to its direction of propagation. Such a disc responds to a very small sound energy, and absorbs only a tiny fraction of that. Most snowflakes have a vaguely disclike structure, derived from the flat hexagonal crystal habit of ice itself, and should turn at right-angles to a suitable sound beam. Furthermore, a disc tends to fall obliquely, gliding unstably sideways parallel to its diameter. So a beam of sound briefly pulsed from the Snow-steerer should turn all the snowflakes in its vicinity parallel to its diverging wave-front. They will then glide slantwise out of the beam, away from the Snow-steerer.

The effect will be sadly transient. The asymetry of the snowflakes and the instability of their glide will soon randomize their fall again. To stabilize their orientation and keep them on their glide-path out of its sound beam, the Snow-steerer will have to sound repeatedly or even continuously. Mercifully, snowflakes are small enough to be orientated by inaudibly ultrasonic wavelengths, so even a very loud snow-steering sound would not annoy a human population. (Bats might be upset, but they have no business to be flying in a snowstorm anyway.)

The Snow-steerer will revolutionize our battles with winter weather. Even in the fiercest snowstorm, the ground around it will remain effortlessly clear. Set along the central barriers of motorways, Snowsteerers will keep the traffic safely flowing. Installed in the middle of a football field, they will repel the snow away from the pitch and onto the spectators. Conversely, set around a ski-slope, they will concentrate all the local snowfall onto the slope. Desperate slope-owners cursing the greenhouse effect will be able to make the best use of their fast-wasting assets. And by subtle control or programming of a Snowsteering array, an ingenious sculptor might create the world's first self-accumulating snowman.

David Jones 